\title{
Construction of Physically Realizable Driving-Point Function From Measured Frequency Response Data on a Model Winding
}

\author{
K. Ragavan and L. Satish, Senior Member, IEEE
}

\begin{abstract}
A simple method is described for constructing physically realizable driving-point impedance function from measured frequency response data (i.e., magnitude and phase) on a model winding and a transformer. A unique feature of the proposed method is that it ensures the constructed rational function is always positive-real, thereby guaranteeing synthesis of a physically realizable network every time. This feature could not always be guaranteed by earlier methods. Hence, it was a limitation. The proposed method is demonstrated on a single-layer model winding and the measured terminal characteristics is converted to a lumped parameter ladder network, since this representation is naturally suited to establish a physical mapping between the actual winding and synthesized circuit. So, the need to guarantee physical realizability is evident. Proceeding further, the terminal characteristics of a 315-kVA, 11/6.9-kV transformer is measured and a rational function representation is obtained. However, its realization as a coupled ladder network requires some more work to be done. In summary, it is believed that this proposal is a step towards providing a solution for localization of deformation in actual transformer windings.
\end{abstract}

Index Terms-Driving-point function, frequency response, Hurwitz polynomial, poles and zeros, rational function, transformer winding deformation.

\section{INTRODUCTION}

$\mathbf{O}$ CCURRENCE of overvoltages and short-circuit forces in a power system is natural, inevitable and one of the main causes for power transformer failure. Depending on its severity, these events can result in deformation/displacement of the winding, which subsequently could develop into major faults. Such initial damages can also occur due to unskilled handling and/or rough transportation to the installation site. Over the years, diagnostic and monitoring tools like low-voltage impulse (LVI) testing and frequency response analysis (FRA) have been found to be reasonably effective in detecting such situations [1]-[4]. However, matters pertaining to interpretation of frequency response data leading to fault localization has not attracted enough attention. It is needless to emphasize the practical significance of solving this problem. In the authors' opinion, achieving this would require a suitable mathematical formulation. The foremost step in this endeavor turns out to be:

Manuscript received December 14, 2006; revised July 30, 2007. Paper no. TPWRD-00802-2006.

The authors are with the High Voltage Laboratory, Department of Electrical Engineering, Indian Institute of Science, Bangalore, India (e-mail: satish@hve. iisc.ernet.in).

Digital Object Identifier 10.1109/TPWRD.2008.915815
- based on input impedance measurements (in frequency domain), develop a procedure to construct a physically realizable rational function;

- starting from the constructed rational function, synthesize a lumped parameter ladder network.

Selection of the lumped parameter ladder network for this purpose is a natural choice, as it not only models the lightning impulse behavior adequately, but also, inherently captures in it the physical length of the winding. In other words, the physical continuous winding can be visualized as being mapped onto a set of discrete nodes, starting from line to neutral end. Quite obviously, non-ladder networks are not suitable for addressing the objective of localization.

The task of synthesizing a ladder network, starting from the measured frequency response data, requires that the data be first represented as a rational function of polynomials in "s". When this rational function satisfies certain specific properties, then synthesis of a physically realizable network (i.e., a ladder network in this case) becomes permissible. Else, it is not possible. The property of physical realizability is an essential prerequisite, which can be achieved, if and only if:

- measured data corresponds to driving-point functions (impedance or admittance);

- rational function constructed must be positive-real.

Amongst the above two, the first is easily taken care of during measurements. With availability of many commercial equipment (specifically meant for FRA measurements), the task of measuring magnitude and phase (corresponding to impedance) is a fairly simple exercise. Once data become available, the next step is to obtain a positive-real rational function corresponding to it. Although, many methods have been proposed to convert frequency response data into equivalent rational functions, they could not always guarantee that it is positive-real. A rational function is said to be positive-real, when both of its (numerator and denominator) polynomials are Hurwitz polynomials, i.e., whose coefficients are of the same sign.

Thus, the primary objective of this paper is to convert drivingpoint impedance data (available in frequency domain) into a positive-real rational function in s-domain. This is demonstrated for a simulated case, a model winding, and an actual transformer.

\section{LITERATURE REVIEW}

Literature pertinent to the objective of this paper were examined. Previous efforts can basically be classified into two categories, viz. the following. 
(i) Building circuit models using terminal measurements. Based on terminal measurements on the winding, there have been some efforts to construct circuit models (all of them are non-ladder) of the windings [5]-[8]. In [9], two non-ladder networks were constructed using frequency response data corresponding to a transformer winding before and after introduction of a fault. Although the circuits reproduced the terminal characteristics well, they did not correspond to physically realizable networks, as resistances were negative and the inductance values far too high.

Equivalent circuit model can also be obtained by using modal analysis as described in [10], wherein each natural oscillation of the transformer is allocated a series resonant circuit. Since the resulting network is non-ladder, a direct mapping of the constructed network with transformer winding is not possible and hence the objective of fault localization cannot be addressed by it.

So, utility of these efforts in the present context is remote.

(ii) Approximating terminal characteristics with rational functions. Although there have been some efforts to construct rational function approximation of terminal characteristics (in s-domain) using frequency response data [11]-[13], they were not always physically realizable. For example, when driving-point function constructed is not a ratio of Hurwitz polynomials, then it cannot be represented by a physically realizable network [14]. Nonetheless, these functional models have been very useful in representing terminal behavior of transformers in transient studies.

Thus, from the above, it emerges that a procedure guaranteeing construction of a positive-real functional approximation of terminal characteristics has not been reported, and hence, forms the subject matter of this paper.

\section{PROBLEMS IN EXISTING APPROACHES}

To construct the driving-point impedance function (DPIF), the following quantities are to be measured at each discrete frequency (using a digital oscilloscope and a current probe).

- Amplitude of input voltage, $V_{s}$, and input current, $I_{s}$

- Time delay, $T_{d}$, between input voltage and input current (estimated when waveforms are zero-crossing and having positive slope)

From the above measured quantities, magnitude, $|Z|$, and phase, $\phi$, of the driving-point impedance at the frequency " $f$ " is determined as

$$
\begin{aligned}
|Z| & =V_{s} / I_{s} \\
\phi & =T_{d} \times f \times 360^{\circ} .
\end{aligned}
$$

With the knowledge of magnitude and phase data over a wide range of frequencies, magnitude and phase plots of drivingpoint impedance can be obtained. Initially, for the sake of explanation, consider a simulated DPIF data, as shown in Fig. 1.

For the data corresponding to Fig. 1, built-in function in MATLAB, "invfreqs" (which is the implementation of the method proposed in [11]), was used to determine $Z(s)$. Apart (a)

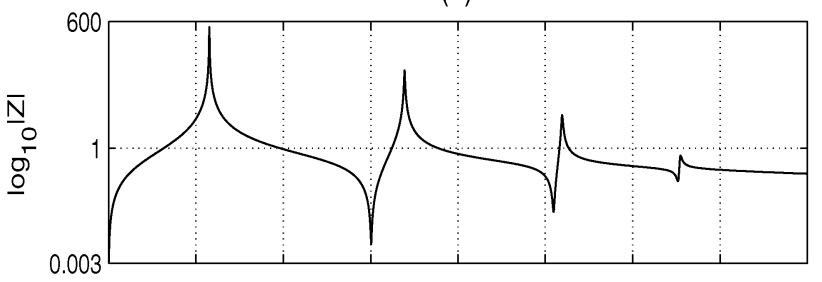

(b)

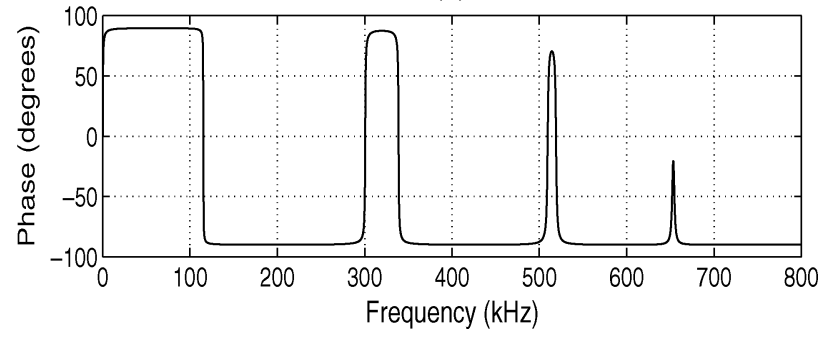

Fig. 1. Simulated driving-point impedance data: (a) magnitude and (b) phase.

from requiring magnitude and phase data at discrete frequencies, orders of the numerator and denominator polynomials are also to be supplied. Since, the data correspond to driving-point impedance, the order of the numerator polynomial is one less than that of the denominator polynomial [15]. A direct use of this function "invfreqs" poses the following problems (linked with correct choice of order of polynomials)-

- By choosing the order of the denominator polynomial less than four (corresponding to the four peaks observable in the magnitude plot), it resulted in rational functions with Hurwitz polynomials. However, the corresponding magnitude and phase plots estimated do not agree with those in Fig. 1. So, it implies that order of the denominator polynomial cannot be less than four.

- When the order of denominator polynomial is four or more, the polynomials constructed are non-Hurwitz and, hence not useful in the present context.

- Further, the process of constructing rational function involves numerical problems due to ill-conditioned matrices and hence the results obtained are unreliable.

- Additionally, existence of noise in FRA data would complicate this matter further.

From the above exercise, it is clear that existing curve-fitting approaches fit a rational function to the frequency response data, but cannot always guarantee its realizability as a network. Hence, there arises a need to devise a suitable method for constructing a network function from practical FRA data and furthermore the method should guarantee that the function constructed corresponds to a physically realizable network always.

\section{Proposed Method for Construction of DPIF}

One of the unique properties of driving-point impedance function is that each pole (or its complex-conjugate) alternates with a zero (or its complex-conjugate). As a result, peaks and troughs in the magnitude response get arranged such that every peak is followed by a trough and vice-versa. This feature is obvious from Fig. 1. Such an orderliness of peaks and troughs permits use of existing relations pertinent to a parallel (or 
series) circuit comprising a resistance, an inductance and a capacitance, for determining the poles and zeros [15].

In [16], it is shown that the driving-point impedance function for the lumped parameter ladder network model consisting of " $n$ " sections, with its neutral being grounded, has $\{n$ pairs of complex-conjugate poles, $(n-1)$ pairs of complex-conjugate zeros and one real zero\}. In practice, all the natural frequencies of the winding may not be well-pronounced in the magnitude frequency plot due to various reasons viz. higher damping due to skin effect, pole-zero cancellation, etc. Suppose, if the frequency response data exhibits only " $m$ " peaks (where, $m<n$ ), and since in these plots, every peak/trough signifies a pair of complex-conjugate poles/zeros, the driving-point impedance function to be constructed would be of the following form [18]:

$$
Z(s)=\frac{\beta_{1}(s-\tau) \prod_{i=1}^{m-1}\left(s-z_{i}\right)\left(s-z_{i}^{*}\right)}{\prod_{i=1}^{m}\left(s-p_{i}\right)\left(s-p_{i}^{*}\right)}
$$

where

$$
\begin{array}{ll}
\beta_{1} & \text { scaling factor; } \\
\tau & \text { real zero; } \\
z_{i}, z_{i}^{*} & \text { complex conjugate zero-pair; } \\
p_{i}, p_{i}^{*} & \text { complex conjugate pole-pair. }
\end{array}
$$

Complex zeros and poles in (2) are of the form

$$
\begin{aligned}
& z_{i}=-\tau_{i} \pm j \psi_{i}, \quad \forall i=1, \ldots, m-1 \\
& p_{i}=-\sigma_{i} \pm j \omega_{i}, \quad \forall i=1, \ldots, m .
\end{aligned}
$$

Starting with this definition of $\mathrm{Z}(\mathrm{s})$, the proposed method of constructing DPIF using frequency response data (as shown in Fig. 1) is comprised of the following steps.

\section{A. Determination of Poles}

Poles of DPIF can be determined using the magnitude and phase plots shown in Fig. 1. The angular frequencies at which magnitude of the impedance approaches the peak value and the phase simultaneously changes from positive to negative, are identified and designated as $\omega_{0, i}$, i.e., undamped open-circuit natural frequencies (ocnf) of the system considered. Real and imaginary parts of the poles are expressed as [15]

$$
\sigma_{i}=\frac{\omega_{0, i}}{2 Q} \quad \text { and } \quad \omega_{i}=\sqrt{\omega_{0, i}^{2}-\sigma_{i}^{2}}
$$

where $Q$ represents the quality factor of the circuit at $\omega_{0, i}$. For $Q \gg 1$ (which is always satisfied in the present context), the above expressjons_become

$$
\sigma_{i} \approx \frac{\omega_{h, i} \_\omega_{l, i}}{2}, \quad \omega_{i} \approx \omega_{0, i} ; \quad \forall i=1, \ldots, m
$$

where $\omega_{l, i}$ and $\omega_{h, i}$ represent the lower and higher 3 -dB frequencies, respectively, in the neighborhood of the undamped ocnf $\omega_{0, i}$. That is, at those frequencies, magnitude of the driving-point impedance is $3 \mathrm{~dB}$ less than that of the magnitude at $\omega_{0, i}$. For determining those $3-\mathrm{dB}$ frequencies, the region around every $\omega_{0, i}$ is considered in the magnitude plot [Fig. 1(a)]. Once the 3-dB frequencies are known, the real and imaginary parts of the pole $p_{i}$ can be determined using (4). Thus, all the poles corresponding to the peaks observed in the magnitude plot [Fig. 1(a)] of driving-point impedance can be determined.

\section{B. Determination of Complex Zeros}

All complex zeros can be estimated by following a similar procedure as described for determining the poles. The angular frequencies at which the impedance approaches the trough in Fig. 1(a) and simultaneously the phase changes from negative to positive in Fig. 1(b) are identified and are denoted as $\psi_{0, i}$, i.e., undamped short-circuit natural frequencies (scnf) of the system considered. If $\psi_{l, i}$ and $\psi_{h, i}$ represent the lower and higher 3-dB frequencies corresponding to which the magnitude is $3 \mathrm{~dB}$ more than the value at $\psi_{0, i}$, then the real and imaginary parts of the complex zeros can be determined as,

$$
\tau_{i} \approx \frac{\psi_{h, i}-\psi_{l, i}}{2}, \quad \psi_{i} \approx \psi_{0, i} ; \quad \forall i=1, \ldots, m-1 .
$$

Thus, all the complex zeros corresponding to the troughs in the magnitude plot [Fig. 1(a)] of driving-point impedance can be determined.

\section{Determination of Real Zero}

Real zero $(\tau)$ can be determined either by using the magnitude plot [Fig. 1(a)] or by considering the low-frequency equivalent circuit. Application of one of these methods can be decided based on the closeness of the real zero to the imaginary axis of s-plane. That is, if the real zero is close to the imaginary axis, a low-frequency equivalent circuit can be used and if it is far away from the imaginary axis, magnitude plot Fig. 1(a) can be utilized. Both these methods for determining real zero are explained here.

1) Magnitude Plot: As the real zero lies close to the imaginary axis compared to other poles and zeros, the region around the zero frequency in the magnitude plot of the driving-point impedance is considered. As this first-order zero is followed by a peak, magnitude of the driving-point impedance increases from $R_{\mathrm{dc}}$, equivalent dc resistance as seen by the input terminals. The frequency $f_{1}$ (in the neighborhood of $0 \mathrm{~Hz}$ ) at which magnitude of the impedance is $3 \mathrm{~dB}$ more than that of the magnitude at 0 $\mathrm{Hz}$ is identified, that is

$$
Z\left(f_{1}\right)=\sqrt{2} R_{\mathrm{dc}}
$$

Hence, the real zero would be at, $\tau=-2 \pi f_{1}$.

If $f_{1}$ is close to $f_{\text {probe }}$ (lower-cutoff frequency of the current probe used for current measurement) and lies in the low-frequency region, then determination of $f_{1}$ using the magnitude plot may not be accurate as the measurements carried out in that frequency range would be unreliable. In such a case, a low-frequency equivalent circuit can be utilized for determining the real zero and is explained below.

2) Low-frequency Equivalent Circuit: The driving-point impedance at low frequencies is

$$
\lim _{s \rightarrow 0} Z(s)=R_{\mathrm{dc}}+s L_{\mathrm{eq}}
$$

where $L_{\text {eq }}$ is the equivalent inductance as seen by the input terminals. 
From the above, the real zero is found to be

$$
\tau=-\frac{R_{\mathrm{dc}}}{L_{\mathrm{eq}}}
$$

Since resistance and inductance $\left(R_{\mathrm{dc}}\right.$ and $\left.L_{\mathrm{eq}}\right)$ can be measured at the input terminals of the winding, real zero can thus be determined without much difficulty.

\section{Estimation of Scaling Factor}

Once the poles and zeros have been determined, the driving-point impedance function can be expressed in the pole-zero form as in (2), in which the scaling factor $\beta_{1}$ is yet to be found. The impedance offered by the equivalent circuit of the winding at zero frequency (i.e., $s=0$ ) is same as $R_{\mathrm{dc}}$ and hence the scaling factor $\beta_{1}$ can be expressed using (2) as

$$
\beta_{1}=R_{\mathrm{dc}} \times \frac{\prod_{i=1}^{m}\left(-p_{i}\right)\left(-p_{i}^{*}\right)}{(-\tau) \prod_{i=1}^{m-1}\left(-z_{i}\right)\left(-z_{i}^{*}\right)} .
$$

Since poles and zeros have already been determined, it becomes straightforward to find the value of scaling factor.

\section{E. Guaranteeing Physical Realizability}

From (2)-(5), it is clear that the DPIF to be constructed using the proposed method would always have its zeros and poles in the left half of s-plane. That is, polynomials of DPIF would have positive coefficients only. Thus the methodology ensures the polynomials of DPIF to be Hurwitz and this feature was not afforded by earlier approaches. Because of this feature, the constructed DPIF is always guaranteed to correspond to a physically realizable network/system.

Note: Since both zeros and poles of driving-point functions are the natural frequencies of the system, their determination by exciting the system becomes possible, whereas zeros of transfer function are in no way related to the natural frequencies of the system [17] and hence their determination by exciting the system is not possible. Therefore, in this work, a driving-point function has been chosen in contrast to the transfer function.

\section{RESULTS AND DISCUSSION}

The principle of the proposed method is illustrated by initially considering a simulated data set (magnitude and phase as in Fig. 1) and thereafter measured FRA data are considered.

\section{A. Simulation Results}

For the simulated FRA data in Fig. 1 pertaining to a ladder network, the steps involved in constructing DPIF are explained below.

a) Complex poles and zeros: In Fig. 1, it is seen that there are four peaks and three troughs. All the frequencies at which peaks and troughs occur are identified and are designated as $f_{0}^{p_{i}}$ and $f_{0}^{z_{i}}$ respectively. Then, $-3 \mathrm{~dB}$ frequencies $\left\{f_{l}^{p_{i}}, f_{h}^{p_{i}}\right\}$ corresponding to every peak frequency $f_{0}^{p_{i}}$ and $+3 \mathrm{~dB}$ frequencies $\left\{f_{l}^{z_{i}}, f_{h}^{z_{i}}\right\}$ corresponding to every trough frequency $f_{0}^{z_{i}}$ are determined. Substituting the values of these frequencies in (4) and (5), poles and zeros can be determined and are presented in Table I and Table II, respectively.
TABLE I

ESTIMATION OF POLES (W.R.T. FIG. 1)

\begin{tabular}{c|c|c|c|c}
\hline \hline$i$ & $\begin{array}{c}f_{0}^{p_{i}} \\
(\mathrm{kHz})\end{array}$ & $\begin{array}{c}f_{l}^{p_{i}} \\
(\mathrm{kHz})\end{array}$ & $\begin{array}{c}f_{h}^{p_{i}} \\
(\mathrm{kHz})\end{array}$ & $\begin{array}{c}p_{i}=\sigma_{i} \pm j \omega_{i} \\
(\mathrm{M} . n \mathrm{neper} / \mathrm{s} \pm j \mathrm{M.rad} / \mathrm{s})\end{array}$ \\
\hline \hline 1 & 115.200 & 115.080 & 115.440 & $0.0011 \pm j 0.7238$ \\
2 & 338.800 & 338.370 & 339.250 & $0.0028 \pm j 2.1287$ \\
3 & 519.200 & 518.470 & 520.170 & $0.0053 \pm j 3.2622$ \\
4 & 654.800 & 653.760 & 658.690 & $0.0155 \pm j 4.1142$ \\
\hline
\end{tabular}

TABLE II

ESTIMATION OF COMPLEX ZEROS (W.R.T. FIG. 1)

\begin{tabular}{c|r|c|c|c}
\hline \hline$i$ & $\begin{array}{r}f_{0}^{z_{i}} \\
(\mathrm{kHz})\end{array}$ & $\begin{array}{c}f_{l}^{z_{i}} \\
(\mathrm{kHz})\end{array}$ & $\begin{array}{c}f_{h}^{z_{i}} \\
(\mathrm{kHz})\end{array}$ & $\begin{array}{c}z_{i}=-\tau_{i} \pm j \psi_{i} \\
(\mathrm{M} . \mathrm{neper} / \mathrm{s} \pm j \mathrm{M.rad} / \mathrm{s})\end{array}$ \\
\hline \hline 1 & 300.600 & 300.150 & 301.030 & $0.0028 \pm j 1.8887$ \\
2 & 509.400 & 508.520 & 510.220 & $0.0053 \pm j 3.2007$ \\
3 & 651.800 & 648.020 & 652.930 & $0.0154 \pm j 4.0954$ \\
\hline
\end{tabular}

b) Real zero: It can be determined using (8) and is found to be, $\tau=-R_{\mathrm{dc}} / L_{\mathrm{eq}}=-\left(3.2 \times 10^{-3} / 1.7897\right)=$ -0.0018 M.neper/s.

c) Scaling factor: It can be determined using (9) and is found to be $\beta_{1}=1.2488$.

d) Polynomials of constructed DPIF: Numerator and denominator polynomials $(N(s)$ and $D(s))$ of DPIF are given as follows:

$$
\begin{aligned}
N(s)= & 1.2488\left(s^{7}+0.0488 s^{6}+30.5843 s^{5}+0.8474 s^{4}\right. \\
& +268.1972 s^{3}+3.1960 s^{2}+612.9248 s \\
& +1.0959) \\
D(s)= & s^{8}+0.0494 s^{7}+32.6252 s^{6}+0.9490 s^{5}+321.8934 s^{4} \\
& +4.4454 s^{3}+976.1467 s^{2}+3.5802 s+427.6785 .
\end{aligned}
$$

It is evident from the above that all the coefficients of polynomials have the same sign.

e) FRA plots with constructed DPIF: Using the constructed polynomials, magnitude and phase are plotted by incrementing the values of $s$ (where $s=j 2 \pi f$ ) and are shown in Fig. 2. Comparing the estimated magnitude and phase with Fig. 1 clearly shows that the match is excellent. This was expected, as it is a simulated data wherein noise is absent.

\section{B. Experimental Results}

The proposed method is validated with practical FRA data obtained by making measurements on: 1) a single-layer model winding and 2) an actual two-winding transformer.

1) FRA on a Model Winding: The model winding consisted of one single-layer (18 SWG insulated wire) wound on an insulated air-core former of diameter $200 \mathrm{~mm}$. The winding had 200 turns and taps were provided after every 20 turns. Using these taps, series and shunt capacitances were connected externally to represent continuous-disc winding, such that: 1) initial voltage distribution constant, $\alpha=7.4833$ and 2) effective value of shunt capacitance to ground, $C_{g, e f f}=5.6 \mathrm{nF}$. 
(a)

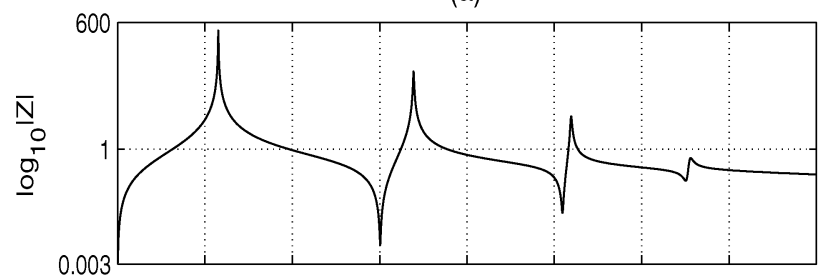

(b)

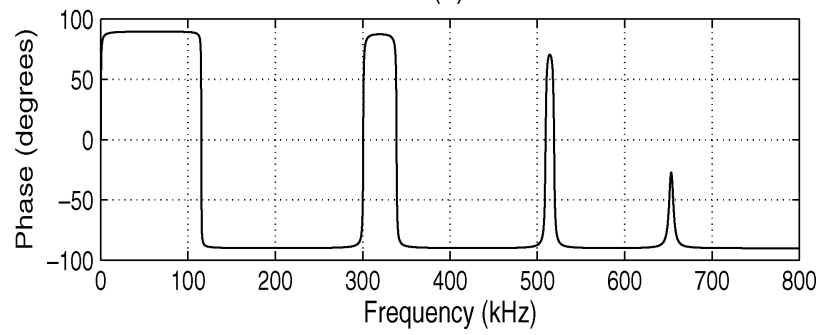

Fig. 2. Estimated driving-point impedance corresponding to simulated data: (a) magnitude and (b) phase.

The following quantities viz. $R_{\mathrm{dc}}=8 \Omega, L_{\mathrm{eq}}=6.98 \mathrm{mH}$ were measured using an LCR bridge. Then FRA is performed on a model winding and some important details of the measurement are as follows.

- A current probe with a bandwidth of $450 \mathrm{~Hz}-60 \mathrm{MHz}$ was employed, selecting a sensitivity of $2 \mathrm{~mA} / \mathrm{mV}$.

- A manual sweep of the frequency was performed along with averaging technique to accurately acquire the input voltage and current waveforms using a digital oscilloscope.

- The incremental frequency was chosen as small as $200 \mathrm{~Hz}$ in the regions corresponding to the peaks and troughs so as to measure them accurately.

- Built-in function in the oscilloscope to measure "time-delay" between two waveforms was used to estimate phase.

The corresponding FRA plots are shown in Fig. 3.

a) Complex poles and zeros: All complex poles and zeros are determined as discussed in Section IV and are listed in Table III and Table IV, respectively.

b) Real Zero: It is found to be $\tau=-R_{\mathrm{dc}} / L_{\mathrm{eq}}=-(8 \times$ $\left.10^{-3} / 6.98\right)=-0.001146$ M.neper $/ \mathrm{s}$

c) Scaling factor: $\beta_{1}=1.3223$.

d) Polynomials of constructed DPIF: Numerator and denominator polynomials of DPIF are given as follows:

$$
\begin{aligned}
N(s)= & 1.3223\left(s^{9}+0.3307 s^{8}+14.0989 s^{7}+2.7623 s^{6}\right. \\
& +60.9120 s^{5}+5.7243 s^{4}+86.9116 s^{3} \\
& \left.+2.3646 s^{2}+27.4406 s+0.0313\right) \\
D(s)= & s^{10}+0.4192 s^{9}+16.3774 s^{8}+4.2570 s^{7} \\
& +85.8872 s^{6}+12.0224 s^{5}+163.4683 s^{4} \\
& +9.4946 s^{3}+90.8031 s^{2}+1.2413 s+5.1683 .
\end{aligned}
$$

It is again observed that the coefficients of both polynomials have the same sign.

e) FRA plots with constructed DPIF: Using the constructed polynomials, magnitude and phase are plotted by sweeping the values of $s$ and are shown in Fig. 4 . A comparison of the estimated magnitude and phase (a)

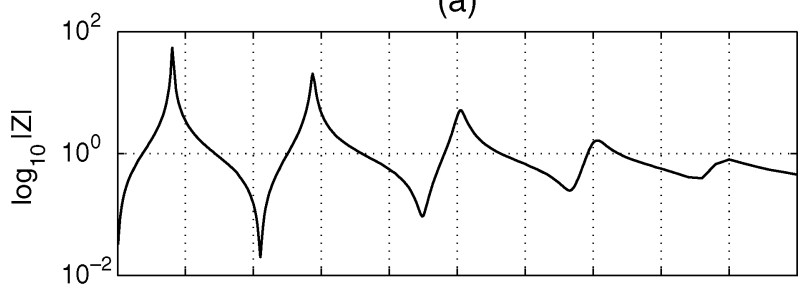

(b)

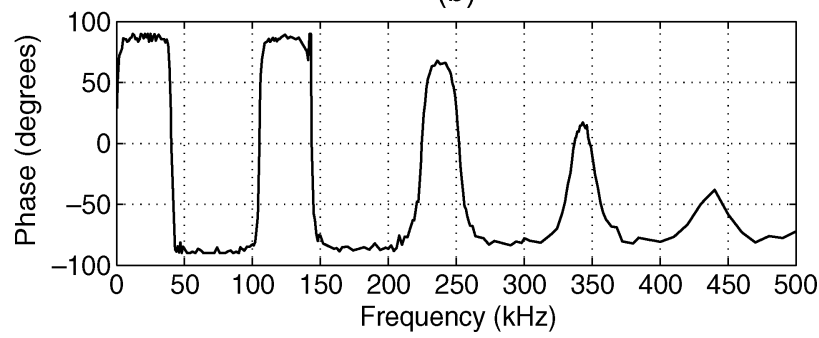

Fig. 3. Measured driving-point impedance on a model winding: (a) magnitude and (b) phase.

TABLE III

ESTIMATION OF POLES (W.R.T. FIG. 3)

\begin{tabular}{c|c|c|c|c}
\hline \hline$i$ & $\begin{array}{c}f_{0}^{p_{i}} \\
(\mathrm{kHz})\end{array}$ & $\begin{array}{c}f_{l}^{p_{i}} \\
(\mathrm{kHz})\end{array}$ & $\begin{array}{c}f_{h}^{p_{i}} \\
(\mathrm{kHz})\end{array}$ & $\begin{array}{c}p_{i}=\sigma_{i} \pm j \omega_{i} \\
(\mathrm{M} . n \mathrm{neper} / \mathrm{s} \pm j \mathrm{M.rad} / \mathrm{s})\end{array}$ \\
\hline \hline 1 & 40.300 & 39.770 & 41.300 & $0.0048 \pm j 0.2532$ \\
2 & 143.500 & 142.380 & 145.070 & $0.0085 \pm j 0.9016$ \\
3 & 252.000 & 249.180 & 256.320 & $0.0224 \pm j 1.5834$ \\
4 & 354.000 & 347.020 & 363.670 & $0.0523 \pm j 2.2242$ \\
5 & 450.000 & 436.310 & 475.010 & $0.1216 \pm j 2.8274$ \\
\hline
\end{tabular}

TABLE IV

ESTIMATION OF COMPLEX ZEROS (W.R.T. FIG. 3)

\begin{tabular}{c|c|c|c|c}
\hline \hline$i$ & $\begin{array}{r}f_{0}^{z_{i}} \\
(\mathrm{kHz})\end{array}$ & $\begin{array}{c}f_{l}^{z_{i}} \\
(\mathrm{kHz})\end{array}$ & $\begin{array}{c}f_{h}^{z_{i}} \\
(\mathrm{kHz})\end{array}$ & $\begin{array}{c}z_{i}=-\tau_{i} \pm j \psi_{i} \\
(\mathrm{M.neper} / \mathrm{s} \pm j \mathrm{M.rad} / \mathrm{s})\end{array}$ \\
\hline \hline 1 & 105.100 & 104.580 & 105.710 & $0.0035 \pm j 0.6604$ \\
2 & 224.000 & 221.100 & 227.010 & $0.0186 \pm j 1.4074$ \\
3 & 332.000 & 323.370 & 337.870 & $0.0456 \pm j 2.0860$ \\
4 & 430.000 & 405.010 & 435.920 & $0.0971 \pm j 2.7018$ \\
\hline
\end{tabular}

with those measured (in Fig. 3) shows that the match is excellent.

2) FRA on a Transformer: FRA was performed on the $11-\mathrm{kV}$ winding of a two-winding transformer $(315 \mathrm{kVA}, 11 / 6.9 \mathrm{kV})$ with its neutral grounded, while the other winding was shortcircuited and grounded. FRA plots are shown in Fig. 5.

Steps involved in constructing the DPIF are as follows.

(i) The peaks and troughs in the magnitude plot are identified manually. For clarity, the peaks are marked in Fig. 5. This manual procedure can be suitably automated at a later stage, if required. 
(a)

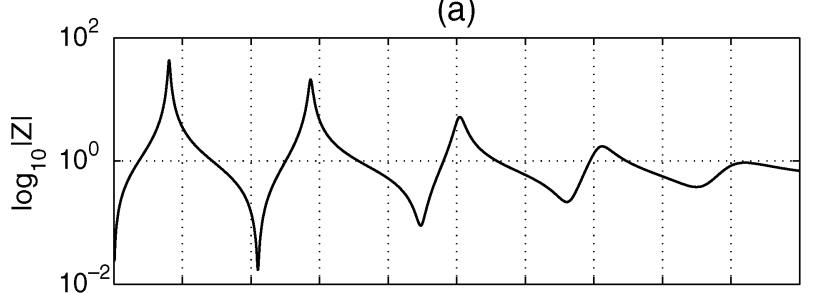

(b)

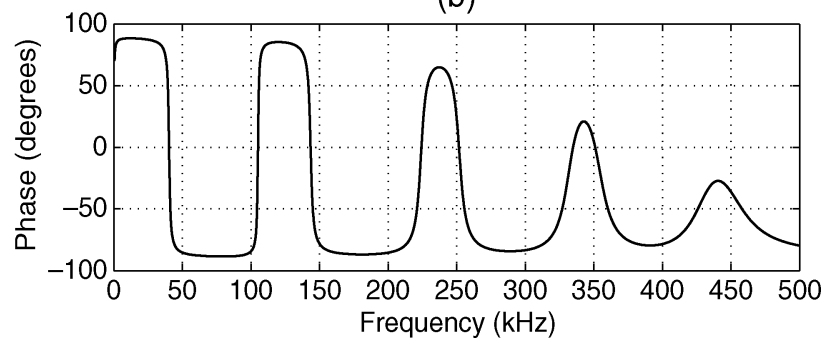

Fig. 4. Estimated driving-point impedance corresponding to model winding: (a) magnitude and (b) phase.

(a)

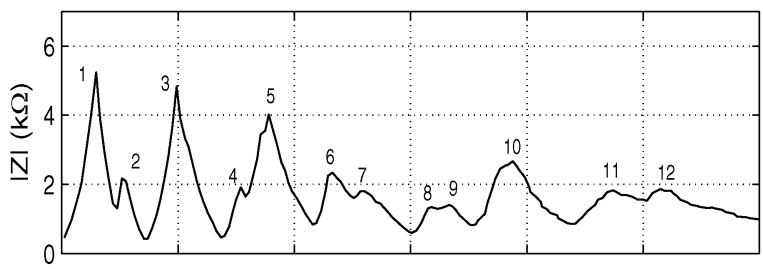

(b)

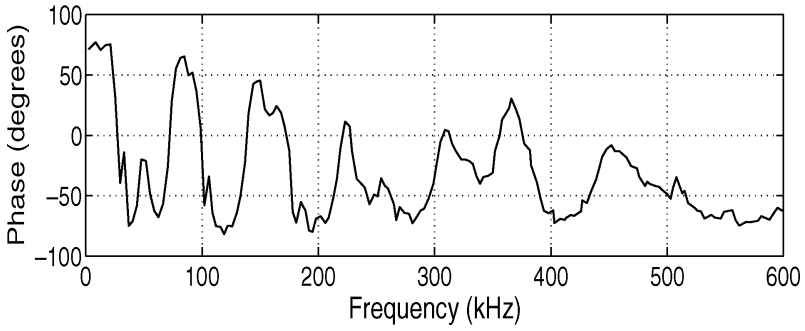

Fig. 5. Measured driving-point impedance on a $315-\mathrm{kVA}, 11 / 6.9-\mathrm{kV}$ transformer: (a) magnitude and (b) phase.

(ii) When the $\pm 3 \mathrm{~dB}$ point corresponding to a peak/trough does not physically exist on the magnitude plot, then it can be estimated approximately as follows.

- In some instances, when one of the 3-dB point is unavailable, then it can be approximated by employing symmetry with respect to the peak. For example, corresponding to the peak marked " 4 ", the upper $-3 \mathrm{~dB}$ point is found by symmetry using the lower $-3 \mathrm{~dB}$ point which exists on the plot.

- When both the upper and lower 3-dB point corresponding to a peak/trough does not physically exist on the magnitude plot, a linear extrapolation (on either side of the peak/trough) is used to estimate the \pm 3 $\mathrm{dB}$ points, wherever necessary. This situation usually occurs when two poles lie very close to each other, for instance, poles marked " 8 \& 9".

(iii) The measured values of $R_{\mathrm{dc}}$ and $L_{\mathrm{eq}}$ are $2.2 \times 10^{-3} \mathrm{k} \Omega$ and $22.4 \mathrm{mH}$, respectively. Hence, real zero, $\tau=$ $-9.8214 \times 10^{-5}$ M.neper/s.
TABLE V

COEFFICIENTS OF DPIF POLYNOMIALS (W.R.T. FIG. 5)

\begin{tabular}{l|r|r}
\hline \hline Order & Numerator $\dagger$ & Denominator \\
\hline \hline & & \\
$s^{24}$ & - & 1 \\
$s^{23}$ & 1 & 1.9675 \\
$s^{22}$ & 1.3183 & 43.5670 \\
$s^{21}$ & 37.8862 & 72.2220 \\
$s^{20}$ & 42.7626 & 789.4306 \\
$s^{19}$ & 596.0294 & 1100.6059 \\
$s^{18}$ & 571.3262 & 7817.6882 \\
$s^{17}$ & 5110.8786 & 9115.4370 \\
$s^{16}$ & 4112.9554 & 46747.4822 \\
$s^{15}$ & 26340.3909 & 45117.7677 \\
$s^{14}$ & 17505.9489 & 175858.4650 \\
$s^{13}$ & 84723.2057 & 138211.1606 \\
$s^{12}$ & 45434.1147 & 420133.6230 \\
$s^{11}$ & 170781.1755 & 262481.7659 \\
$s^{10}$ & 71506.1957 & 628133.5154 \\
$s^{9}$ & 210864.2086 & 301380.3746 \\
$s^{8}$ & 65715.3125 & 564511.3191 \\
$s^{7}$ & 151191.6550 & 197884.0338 \\
$s^{6}$ & 32571.8599 & 282529.6542 \\
$s^{5}$ & 56997.6763 & 67053.4953 \\
$s^{4}$ & 7462.0218 & 68366.7100 \\
$s^{3}$ & 9318.2680 & 9711.6522 \\
$s^{2}$ & 567.9705 & 6226.6991 \\
$s^{1}$ & 485.9614 & 409.3840 \\
$s^{0}$ & 0.0477 & 151.7732 \\
\hline
\end{tabular}

$\dagger$ Numerator coefficients have to be multiplied by $\beta_{1}=3.6$

(a)

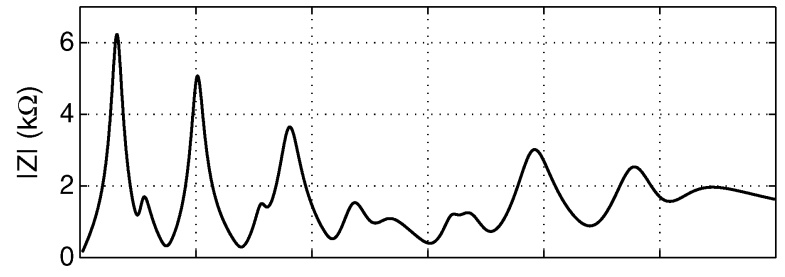

(b)

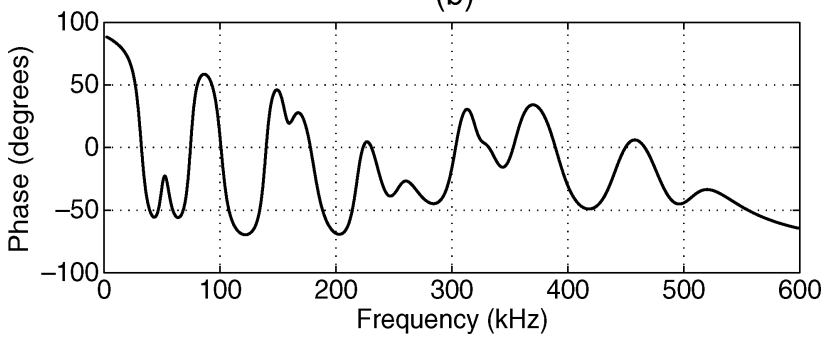

Fig. 6. Estimated driving-point impedance corresponding to $315-\mathrm{kVA}$, 11/6.9-kV transformer: (a) magnitude and (b) phase.

(iv) Scaling factor is found to be $\beta_{1}=3.6$.

By deploying the proposed approach together with the above guidelines, DPIF is constructed and the coefficients are listed in Table V. Further, using this rational function, the magnitude and phase plots are obtained and are presented in Fig. 6.

The following points emerge from the results presented.

- FRA plots obtained using the constructed rational DPIF agree very well with those measured on the transformer. That is, terminal characteristics of the transformer are modeled accurately by the rational function. All the subtle aspects seen in the measured data are well reproduced in the magnitude and phase plots obtained from the constructed DPIF. 
TABLE VI

SELF AND Mutual INDUCTANCES ${ }^{\dagger}$ FOR THE NETWORK IN FIG. 7

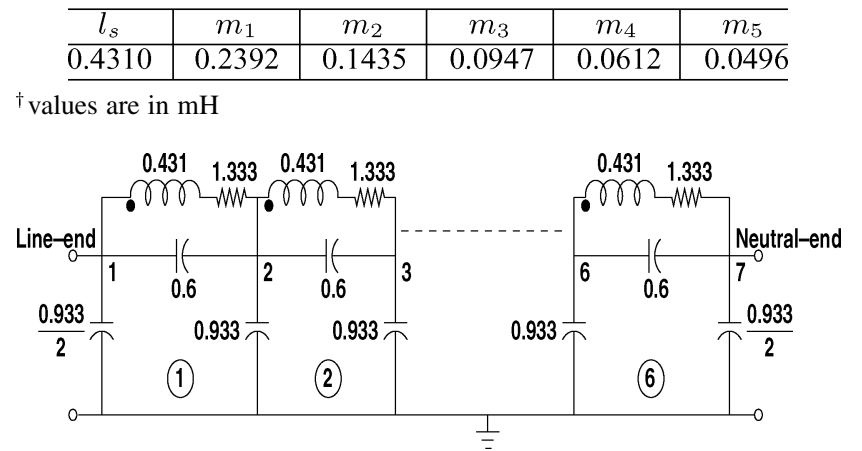

Fig. 7. Synthesized ladder network using constructed DPIF (refer Table VI for inductance values. Resistance, inductance and capacitance are in $\Omega, \mathrm{mH}$, and $\mathrm{nF}$, respectively)

- From the constructed DPIF, it is clear that all coefficients of both the numerator and denominator polynomials are positive (i.e., similar in sign), implying that the constructed DPIF consists of Hurwitz polynomials only.

\section{Physical Realizability of DPIF AS A LADDER NETWORK}

An attempt is made to synthesize a physically realizable ladder network corresponding to the data acquired from the single-layer model winding (whose terminal characteristics is shown in Fig. 3).

Starting from the constructed rational function (described by polynomials in Section V) and deploying the method illustrated in [18] (more details are available in [16]), it is possible to meet the objective. Initially the number of sections in the ladder network to be synthesized is estimated (here, it is 6) and then the elemental capacitances (series capacitance/section: $0.6 \mathrm{nF}$, shunt capacitance/section: $0.933 \mathrm{nF}$ ) are determined.

Since there exists symmetry in the model winding, it is appropriate to consider the self inductance of all the sections as identical and the mutual inductance between sections $k$ and $(k+i)$ can be expressed as

$$
m(k, k+i)=m_{i} ; \quad \forall i=1, \ldots, 5 \text { and } k=1, \ldots,(6-i) .
$$

Self and mutual inductances are estimated iteratively and for brevity, only the results are presented in Table VI.

(Note: In case of no symmetry in the winding disposition, the above expression is not valid and all of the self and mutual inductances are to be estimated similarly.)

The resulting synthesized ladder network is shown in Fig. 7. It faithfully reproduces the same terminal characteristics as measured with the model winding (Fig. 3). Similar such ladder networks can be synthesized corresponding to the cases with the introduction of faults. A comparison of these networks with the network corresponding to the fault-free case reveals the nature, location, and extent of the fault. Several examples illustrating this possibility of mapping the changes in the physical winding in terms of the variations in circuit elements are presented in [18].

It must be noted that the proposed method still has a long way to go, before it can be applied to multiwinding transformers. As a matter of fact, more than one ladder network will have to be synthesized corresponding to each phase and number of windings present. The electrostatic and magnetic couplings within the windings and between the windings are to be considered in the circuit model. Also, some thought has to be given regarding handling non-uniformity, most often present in large transformers, due to interleaving, tap-winding, tertiary winding, and so on, during circuit synthesis. Further, it must be noted that frequency response data have to be limited to about $2 \mathrm{MHz}$ (an optimistic upper limit) while constructing the rational function because such lumped-parameter circuits have an upper bound on the frequency, after which, such circuit representations will no longer be valid. Lastly, the accuracy and resolution with which the frequency response data are acquired will play a vital role in the accuracy of the rational function constructed.

\section{DISCUSSION}

1) The match between the measured and estimated frequency response was observed to be very good in all cases.

2) The formulation is such that the method does not suffer from any numerical problems. This, in fact, was a limitation of the earlier methods.

3) Noise which is usually present in practical data, does not seriously affect the estimated values, as was demonstrated when actual FRA data on a transformer was processed.

4) The constructed $Z(s)$ is always guaranteed to be positive-real. Thus, ensuring physical realizability. Once the system can be physically realized, it enables construction of physical models and measurement of system performance, for example, estimation of voltage distribution in the winding, determination of the transferred voltage at any internal node and hence the severity of the electric stress at different locations of the winding, etc.,

5) For all cases discussed, complete details of the constructed DPIF have been furnished, to enable independent verification of the results.

6) The method described is very general and hence applications can be many irrespective of the field. Perhaps, one possible example could be, in power system studies (to estimate time-domain responses), the method could be employed to represent a transformer (as a module in frequency domain) up to about $2 \mathrm{MHz}$.

\section{CONCLUSION}

A simple and elegant procedure has been proposed to convert the terminal characteristics obtained from a model winding and a transformer into a rational function. As the constructed rational functions consist of Hurwitz polynomials only, its conversion into physical networks is guaranteed. To demonstrate this, a ladder network is synthesized corresponding to frequency response data acquired from a single-layer model winding. It is believed that synthesizing such physically realizable ladder networks corresponding to the terminal characteristics of transformer would be of some help in locating mechanical deformations in it. Although the method has been illustrated for the FRA 
data corresponding to transformer, it is equally applicable to derive a model for any physical system.

\section{REFERENCES}

[1] W. Lech and L. Tyminski, "Detecting transformer winding damage by the low voltage impulse method," Elect. Rev., vol. 179, no. 21, pp. 768-772, Nov. 1966.

[2] E. P. Dick and C. C. Erven, "Transformer diagnostic testing by frequency response analysis," IEEE Trans. Power App. Sys., vol. PAS-97, no. 6, pp. 2144-2153, Nov./Dec. 1978.

[3] R. Malewski and B. Poulin, "Impulse testing of power transformers using the transfer function method," IEEE Trans. Power Del., vol. 3, no. 2, pp. 476-489, Apr. 1988.

[4] T. Leibfried and K. Feser, "Monitoring of power transformers using the transfer function method," IEEE Trans. Power Del., vol. 14, no. 5, pp. 1333-1341, Oct. 1999.

[5] A. O. Soysal, "A method for wide frequency range modeling of power transformers and rotating machines," IEEE Trans. Power Del., vol. 8, no. 4, pp. 1802-1808, Oct. 1993.

[6] R. C. Degeneff, "A general method for determining resonances in transformer windings," IEEE Trans. Power App. Syst., vol. PAS-96, no. 2, pp. 423-430, Mar./Apr. 1977.

[7] P. T. M. Vaessen, "Transformer model for high frequencies," IEEE Trans. Power Del., vol. 3, no. 4, pp. 1761-1768, Oct. 1988.

[8] A. Morched, L. Martf, and J. Ottevangers, "A high frequency transformer model for the EMTP," IEEE Trans. Power Del., vol. 8, no. 3, pp. 1615-1626, Jul. 1993.

[9] D. M. Sofian, Z. D. Wang, and P. Jarman, "Interpretation of transformer FRA measurement results using winding equivalent circuit modeling technique," in Proc. Conf. Electrical Insulation and Dielectric Phenomena (CEIDP 2005), Nashville, TN, Oct. 16-19, 2005, pp. 613-616.

[10] P. Glaninger, "Modal analysis as a means of explaining the oscillatory behaviour of transformers," Brown Boveri Rev., vol. 1-86, pp. 41-49, 1986.

[11] E. C. Levy, "Complex curve fitting," IRE Trans. Autom. Control, vol. AC-4, pp. 37-44, May 1959.

[12] A. H. Whitfield, "Transfer function synthesis using frequency response data," Int. J. Contr., vol. 43, no. 5, pp. 1413-1426, 1986.
[13] B. Gustavsen and A. Semlyen, "Rational approximation of frequency domain responses by vector fitting," IEEE Trans. Power Del., vol. 14 no. 3, pp. 1052-1061, Jul. 1999.

[14] J. Vlach, Computerized Approximation and Synthesis of Linear Networks. New York: Wiley, 1969.

[15] C. A. Desoer and E. S. Kuh, Basic Circuit Theory. New York: McGraw-Hill, 1969.

[16] K. Ragavan, "An Efficient Solution to Generalized Model of a Transformer Winding and Localization of Discrete Changes Based on Measurements," Ph.D. dissertation, High Voltage Laboratory, Dept. Elect. Eng., Indian Inst. Science, Bangalore, India, 2006.

[17] E. A. Guillemin, Synthesis of Passive Networks. New York: Wiley, 1962.

[18] K. Ragavan and L. Satish, "Localization of changes in a model winding based on terminal measurements: Experimental study," IEEE Trans. Power Del., vol. 22, no. 3, pp. 1557-1565, Jul. 2007.

K. Ragavan was born in India in 1976. He received the B.Tech. degree from Pondicherry Engineering College, Pondicherry, India, in 1997, the M.E. degree from Anna University, Chennai, India, in 2002, and the Ph.D. degree from the High Voltage Laboratory, Department of Electrical Engineering, Indian Institute of Science, Bangalore, India, in 2006.

He has been with the GE India Technology Centre Pvt. Ltd., Bangalore, since January 2007.

L. Satish (SM'02) was born in 1964. He received the Ph.D. degree from the Indian Institute of Science (IISc), Bangalore, India, in 1993.

He was a Postdoctoral Fellow with Eidgenössische Technische Hochschule (ETH) Zurich, Zurich, Switzerland, from 1993 to 1995. He joined the Department of High Voltage Engineering, IISc, in 1995, and is currently a Professor in the High Voltage Laboratory, Department of Electrical Engineering, IISc. His research interests include application of signal processing to high-voltage impulse testing, diagnostics, condition monitoring, partial-discharge measurements, pattern recognition, and ADC testing. He was a Visiting Researcher at the High Voltage Institute, Helsinki University of Technology, Helsinki, Finland, in 1998.

Dr. L. Satish is a member of CIGRE WG D1-33. 